\title{
Perspectivas de tratamiento de la alergia al látex: inmunoterapia
}

\section{Perspectives in the treatment of allergy to latex: immunotherapy}

\section{A.I. Tabar, B. Gómez, E. Arroabarren, M. Rodríguez, I. Lázaro, M. Anda}

\section{RESUMEN}

En la actualidad la alergia al látex es un problema importante dada la gravedad de la sintomatología que produce y los grupos de riesgo a los que implica. La evitación completa del látex resulta prácticamente imposible, por lo que en los últimos años se ha estado trabajando intensamente en la estandarización de un extracto con el fin de mejorar no sólo el diagnóstico clíinco sino de poder oferar otra alternativa terapéclíva terapéutica la evitación tal como la inn

Desde 1998 se han comunicado en la literatura aproximaciones puntuales de inmunoterapia con látex con pauta de desensibilización oral (tres pacientes) subcutánea (un paciente) y sublingual (un paciente) En todos los casos la mejoría clínica fue evidente.

En el momento actual, la inmunoterapia subcutánea con látex, aunque se muestra eficaz, es un tratamiento de alto riesgo.

La IT sublingual con látex ha sido recientemente comercializado en España y conocemos su eficacia por los resultados de un ensayo abierto en 26 pacientes adultos. Las expectativas de seguridad se muestran mejores que para la vía subcutánea.

Palabras clave. Látex. Tratamiento. Inmunoterapia subcutánea. Seguridad. Eficacia. Inmunoterapia sublingual.

An. sis. sanit. Navar. 2003; 26 (Supl. 2): 97-102.

\section{ABSTRACT}

At present, allergy to latex is an important problem due to the severity of the symptomatology that it produces and the risk groups involved. Complete avoidance of latex is practically impossible, which is why in recent years there has been intense work on standardizing an extract in order not only to improve its clinical diagnosis but also to be able to improve its clinical diagnosis but also to be able to offer a therapeutic alter
such as immunotherapy.

Since 1998, timely approaches to immunotherapy with latex have been published, with oral desensitization (three patients), subcutaneous (one patient) and sublingual (one patient). In every case there was an evident clinical improvement.

At present, subcutaneous immunotherapy with latex, although efficient, is a high risk treatment.

Sublingual immunotherapy with latex has recently been commercialized in Spain and we know of its efficacy through the results of an open test on 26 adul patients. The safety expectations are shown to be better than those for subcutaneous immunotherapy.

Key words. Latex. Treatment. Subcutaneous immunotherapy. Security. Efficacy. Sublingual immunotherapy.
Sección de Alergología. Hospital Virgen del Camino. Pamplona.

\author{
Correspondencia: \\ Ana Isabel Tabar Purroy \\ Sección de Alergia \\ Hospital Virgen del Camino \\ CS Conde Oliveto \\ Pza. de la Paz s/n \\ 31002 Pamplona \\ Tfno: 948429308 \\ Fax: 948429271
}




\section{INTRODUCCIÓN}

En el momento actual la alergia al látex es un problema importante para el paciente, dada la gravedad de la sintomatología que produce (oscila desde urticaria de contacto, rinoconjuntivitis y/o asma bronquial, hasta anafilaxia) y los grupos de riesgo a los que implica (sanitarios, en los que cumple características de enfermedad ocupacional y multioperados como niños con espina bífida). Además la evitación completa es difícil, si no imposible, dada su ubicuidad.

En los cinco últimos años se ha estado trabajando intensamente en la estandarización de extracto de látex, puesto que de conseguirla, mejoraría el diagnóstico clínico y además podría considerarse la inmunoterapia específica como una alternativa terapéutica.

Ocho trabajos han sido presentados hasta la fecha, de inmunoterapia subcutánea con látex. De los tres primeros, uno está realizado en tres pacientes y los otros dos en un solo paciente y de forma abierta. El resto incluye mayor número de pacientes y/o tiene diseño doble ciego frente a placebo.

La primera aproximación ${ }^{1}$ fue presentada en el Congreso Americano de Alergia en el año 1998, realizándose una desensibilización oral en un estudio abierto sobre tres pacientes asmáticos, con alergia ocupacional al látex. Los autores concluyen que la desensibilización a látex es segura y que mejora de forma notable la sintomatología clínica, produciendo así mismo disminución en el área de los test cutáneos.

La segunda comunicación fue un Allergy $\mathrm{Net}^{2}$ en que se realizó inmunoterapia en una mujer de 31 años, técnico de Radiología, con sintomatología rinoconjuntival al contacto con látex, clínica que evoluciona a urticaria, asociándose en el tiempo anafilaxia con alimentos vegetales. Los autores afirman que el extracto empleado fue seguro y efectivo en la paciente.

Recientemente, Nucera y col$^{3}$ publicaron un caso de inmunoterapia sublingual con extracto de látex en una mujer de 23 años, estudiante de medicina, que presentaba urticaria de contacto, asma y rinitis con la manipulación de guantes. Tras el diagnóstico, la paciente fue sometida a una pauta de desensibilización rápida sublingual con extracto de látex, utilizando como dosis de mantenimiento 5 gotas de una solución que contiene $500 \mathrm{mgr} / \mathrm{ml}$ de látex, una vez al día. Según los autores el tratamiento en esta ocasión también resultó seguro y eficaz.

\section{INMUNOTERAPIA SUBCUTÁNEA \\ CON LÁTEX}

En 1997 se publicó en la revista Allergy la estandarización in vivo e in vitro de un extracto de látex natural validado para diagnóstico. La estandarización biológica del extracto se realiza en unidades biológicas (IR). Un extracto alergénico es titulado a $100 \mathrm{IR} / \mathrm{ml}$ cuando utilizado en prick con lancetas Stalerpoint $®$ y en 30 personas sensibilizadas a este alergeno provoca una pápula de diámetro medio de $7 \mathrm{~mm}$ (media geográfica). El extracto validado en unidades biológicas (IR) muestra una alta sensibilidad (93\%), una alta especificidad (100\%) y buen perfil de seguridad.

La disponibilidad de un extracto de calidad conduce a la realización del primer estudio piloto para la evaluación de la tolerancia y la eficacia de la inmunoterapia específica con látex en pacientes con alergia ocupacional, estudio en fase $2 \mathrm{~B}$ aleatorio, doble ciego, controlado con placebo, efectuado en tres centros hospitalarios, dos de Paris y uno de Marsella, incluyendo 20 pacientes con una pauta rápida de inicio de 48 horas y un tratamiento de mantenimiento de 12 meses $^{5}$.

Los pacientes del ensayo son personal sanitario con una exposición al látex no evitable, con una edad mayor de 18 años y menor de 50, con síntomas de rinitis y/o asma, pruebas cutáneas positivas al látex IgE específica $>0,70 \mathrm{kU} / \mathrm{l}$ y una prueba de provocación conjuntival también positiva.

Se utilizaron extractos alergénicos acuosos de látex, estandarizados, en concentraciones de $0,01 \mathrm{IR} / \mathrm{ml}, 0,1 \mathrm{IR} / \mathrm{ml}, 1$ $\mathrm{I} / \mathrm{ml}, 10 \mathrm{IR} / \mathrm{ml}$ y $100 \mathrm{IR} / \mathrm{ml}$ y como placebo, diluyente con histamina.

Como criterios de evaluación de eficacia se evaluó puntuación de síntomas, pun- 
tuación de medicación y variación del umbral de reactividad conjuntival. Además se valoró la tolerancia del extracto.

En el análisis total del registro de síntomas, el grupo tratado muestra una mejoría marcada comparado con placebo en términos de sintomatología rinoconjuntival y cutánea.

En referencia a la puntuación de medicación en cada grupo (Fig. 1) se observa que el radio del área bajo la curva es de un $21 \%$, por lo que de acuerdo con la literatu$\mathrm{ra}^{6}$ este dato indica alta eficacia de tratamiento con un $80 \%$ de mejoría clínica.
Tal y como se demuestra en la figura 2 , la dosis necesaria para producir síntomas equivalentes en la provocación conjuntival, aumenta en 7 pacientes del grupo activo (7 de 9) frente a 1 de 8 pacientes del grupo placebo $(\mathrm{P}<0,02$ seguridad $)$

La frecuencia de reacciones, tanto locales como sistémicas, en relación con un número total de inyecciones es alta en comparación con la inmunoterapia convencional. Cerca de la mitad de los pacientes que reciben tratamiento activo tienen reacción local desde la primera inyección (Fig 3). Las reacciones sistémicas, tales como rinitis y

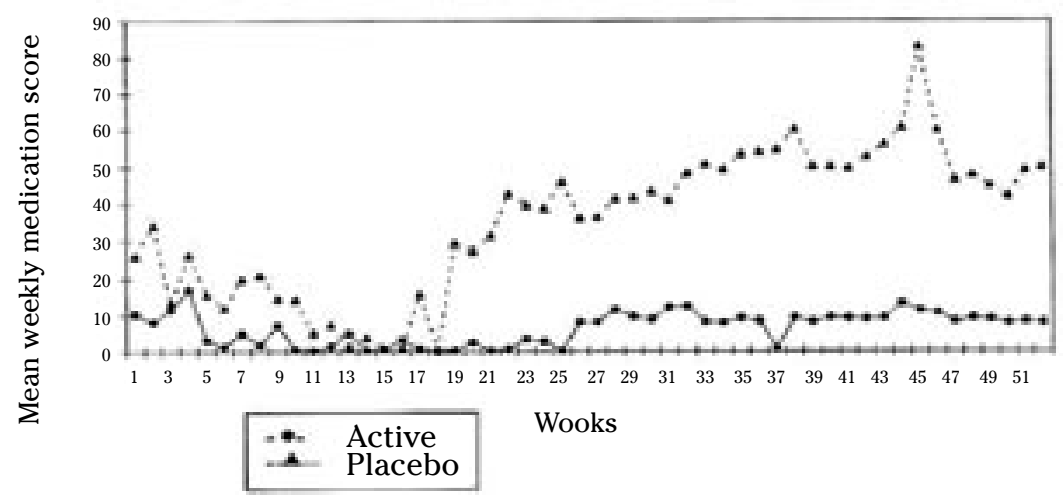

Figura 1. Puntuación de medicación de los grupos activo y placebo. Tomado de Leynadier y col. ${ }^{5}$.
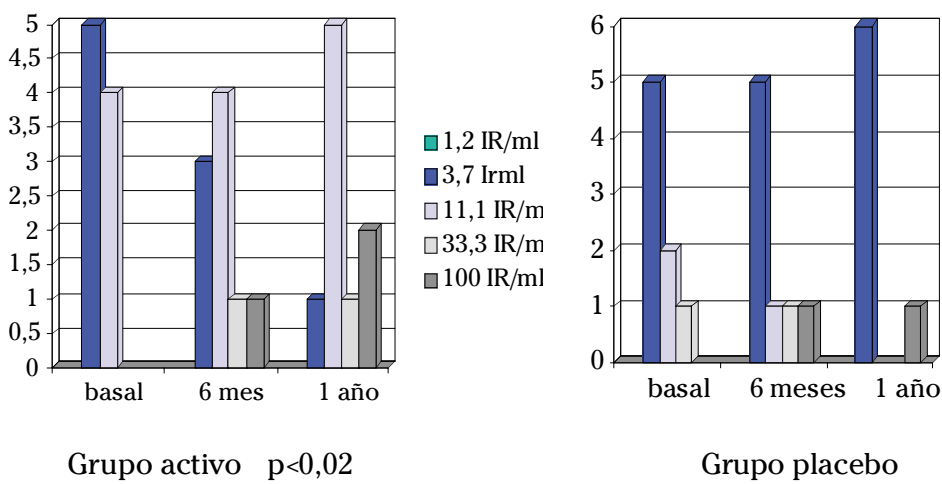

Grupo placebo

Figura 2. Resultados de la provocación conjuntival. Tomado de Leynadier y col. ${ }^{5}$. 


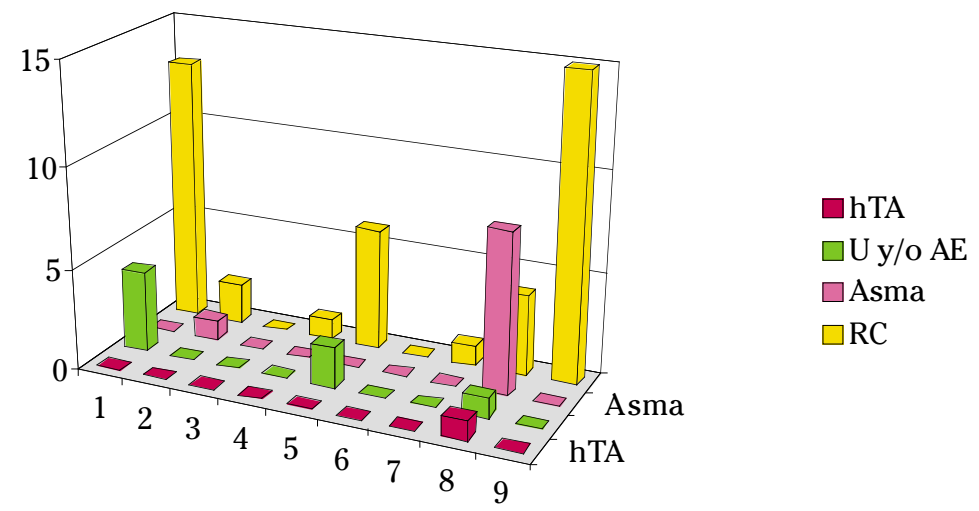

Figura 3. Reacciones sistémicas durante el tratamiernto en los pacientes del grupo activo. Tomado de Leynadier y $\mathrm{col}^{5}$.

conjuntivitis, que se resuelven rápidamente con poca medicación, se registran en los dos grupos, activo y placebo y en el vial de $1 \mathrm{IR} / \mathrm{ml}$. De los pacientes del grupo activo, 4 de los 9 sufren reacciones sistémicas más severas tales como angioedema, urticaria, asma y en un caso hipotensión.

Aunque el número de reacciones adver sas se dispara al insistir una y otra vez en el mismo paciente y tras la primera reacción, en alcanzar la dosis de mantenimiento, la dosis real tolerada en todos los pacientes es de 0,5 IR. Con la siguiente dosis de 1 IR, tres pacientes sufren reacción sistémica, más o menos severa.

Por tanto a la vista de este primer ensayo clínico, puede deducirse que la inmunoterapia frente al látex con este diseño de pauta rápida está lejos del tratamiento ideal. El ensayo, demuestra la eficacia al disminuir la puntuación de medicación y la sintomatología de los pacientes, al menos en términos de conjuntivitis, rinitis y síntomas cutáneos, pero es un tratamiento de alto riesgo en cuanto a seguridad. No obstante, este primer ensayo permite vislumbrar la dosis óptima, dosis que balancea eficacia y seguridad.

En el momento actual nos encontramos en la fase de mantenimiento de un ensayo clínico multicéntrico en el que participa nuestro grupo, con el mismo extracto y algunas variaciones en la pauta de admi- nistración (datos no publicados). El diseño del estudio es también randomizado, doble ciego frente a placebo y la fase de inmunoterapia de inicio en este caso es una pauta rápida de 48 horas, en régimen hospitalario.

Los objetivos de este nuevo ensayo son validar el protocolo anterior, confirmar la eficacia de inmunoterapia frente a látex, mejorar obviamente su seguridad y si es posible determinar la dosis óptima. El primer análisis realizado muestra resultados acordes con los objetivos.

El segundo ensayo clínico de inmunoterapia subcutánea realizado utiliza un extracto de látex estandarizado biológicamente, cuyas características han sido comunicadas recientemente por el grupo ALK-Abelló-España ${ }^{7}$ en el Congreso Americano de Alergia del 2001. Los autores concluyen que el extracto es válido para diagnóstico y tratamiento en pacientes seleccionados con alergia a látex.

Sastre y $\mathrm{col}^{8}$ presentaron en el 2002 en la Reunión Anual de la Sociedad Aragonesa de Alergología los resultados del primer estudio doble ciego frente a placebo con inmunoterapia con látex en pacientes alérgicos con este extracto referenciado recientemente. Los autores incluyeron 24 pacientes sensibilizados a látex y con sintomatología ocupacional. Para diagnóstico de alergia respiratoria se realizó una prueba de pro- 
vocación inhalativa, en cámara cerrada de 7 $\mathrm{m}^{3}$, que contiene aire ultrafiltrado y donde el paciente sacude un par de guantes de látex del mismo lote cada 3 minutos. El tiempo de exposición se incrementa progresivamente $(3,5,15,20$ y 60 minutos) hasta una caída en el VEMs del $20 \%$ y/o hasta un tiempo acumulado de 114 minutos.

En el caso de urticaria de contacto, se realizó un test de uso de guante y un test de frotamiento," rubbing", usando de control un guante de vinilo. Se evaluó la aparición de eritema, prurito y habones a los 15 y 60 minutos.

El test de provocación inhalativa y los test cutáneos se realizaron antes y al final del tratamiento. También se realizó un seguimiento con cartillas de síntomas y uso de medicación durante todo el tratamiento. Dieciocho pacientes presentaron rinitis y conjuntivitis, 14 asma y 13 urticaria de contacto.

El tratamiento consistió en una fase de dosis crecientes de 14 semanas con 18 inyecciones, (las dos primeras visitas en una pauta cluster), durante un total de seis meses. La dosis de mantenimiento que se realizó cada dos semanas, teórica, fue de 5 HEP. Dieciséis pacientes recibieron tratamiento activo y 8 placebo.

Un $62 \%$ de los pacientes alcanzó la dosis máxima. La media de dosis tolerada fue de 3,4 HEP. De 578 dosis, en 41 (7,1\%) hubo algún efecto adverso ( Fig. 4)

Se contabilizaron 21 reacciones sistémicas inmediatas (5,7\% de las dosis) y 10 (2,6\% de las dosis) sistémicas tardías en el grupo actual, siendo más frecuentes en los pacientes con manifestaciones respiratorias $(\mathrm{P}<0,05)$. Todas las reacciones sistémicas respondieron bien al tratamiento.

Al final de los seis meses de tratamiento, se evidenció que el ensayo de líneas paralelas de la titulación de pruebas cutáneas con el extracto de látex en los pacientes que recibieron inmunoterapia, tenían un índice de respuesta cutánea 8,9 veces menor $(\mathrm{P}<0,01)$.

La puntuación de síntomas de la prueba de frotamiento y la prueba de uso de guantes de látex en los pacientes que recibieron tratamiento activo, fue menor que en los que recibieron placebo $(\mathrm{P}<0,05)$.

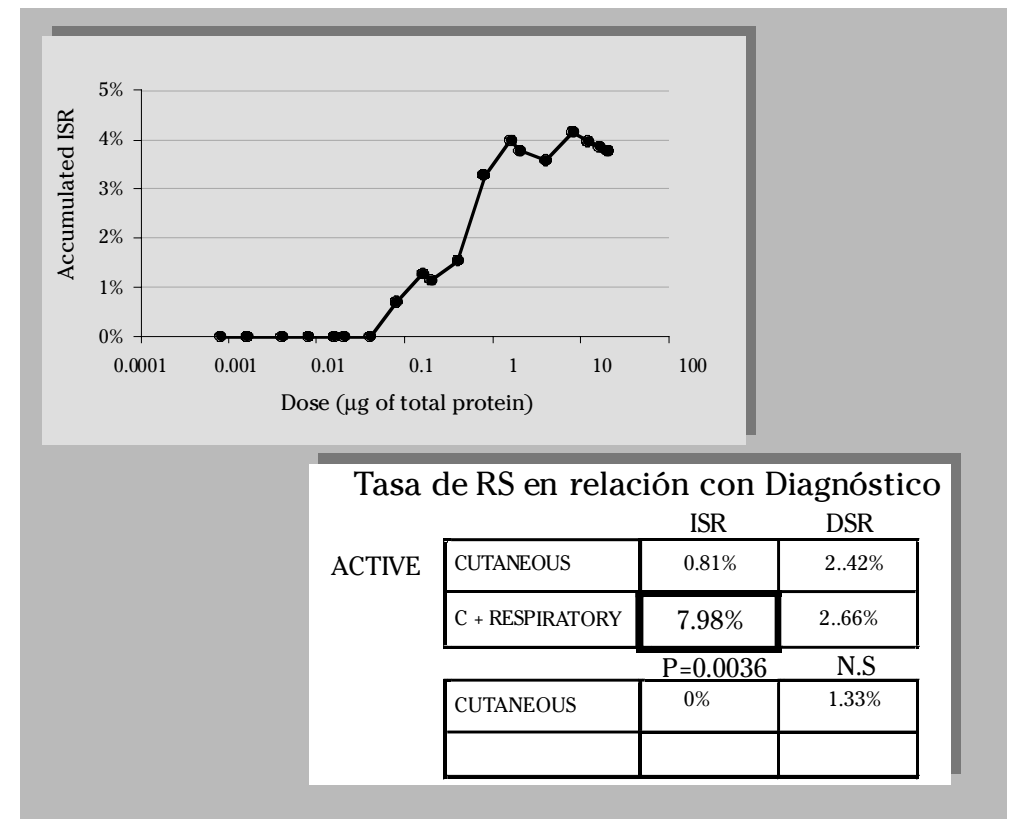

Figura 4. Perfil de tolerancia de la inmunoterapia con látex en el trabajo de Sastre y col's. 
Los autores concluyen que el tratamiento es un tratamiento de riesgo. Que la dosis de $1 \mathrm{mg}$ puede ser una dosis razonable y que la mejoría más marcada se refleja en los síntomas cutáneos.

Aunque de acuerdo con las recomendaciones y perspectivas publicadas por el grupo de trabajo internacional del látex ${ }^{9}$ se requieren más ensayos clínicos y con mayor número de pacientes, las dos aproximacio nes realizadas mediante dos ensayos clínicos, metodológicamente bien estructurados, son esperanzadoras y nos permiten afirmar que la inmunoterapia de látex, en las condiciones comentadas, es un tratamiento eficaz, aunque por el momento su uso debe ser restringido al medio hospitalario.

\section{INMUNOTERAPIA SUBCUTÁNEA CON LÁTEX}

La inmunoterapia sublingual (SLIT) con látex ha sido recientemente comercializada en España. Sólo dos grupos clínicos han comunicado su experiencia hasta la fecha. Patriarca y cols ${ }^{10,11}$ administran el tratamiento a 12 pacientes, sin registrarse reacciones y tolerando tras el mismo la utilización de guantes.

Sastre y $\mathrm{col}^{8}$ realizan un ensayo abierto en 26 pacientes adultos alérgicos al látex. Todos los pacientes alcanzan las dosis de mantenimiento, el test de uso de guantes es mejor tolerado y también se obtiene mejoría en el "rubbing test". El 24\% de las dosis administradas producen reacciones adversas, aunque sólo el $10 \%$ de los casos requirieron tratamiento.

Aunque los diseños de los ensayos no son tan estrictos como en la vía subcutánea, podemos concluir que el tratamiento se muestra más seguro que el parenteral y que al menos en los síntomas cutáneos resulta eficaz.

Por tanto, estamos ante una nueva posibilidad terapéutica para los enfermos con alergia al látex, pacientes en los que hasta la fecha y tras el diagnóstico sólo les podíamos recomendar tratamiento sintomático y medidas de evitación, medidas en muchos casos insuficientes a la vista de la ubicuidad del alergeno.

\section{BIBLIOGRAFÍA}

1. Toci G, Shah S, Al-Fagih A, Beezold D, McGeady SJ, Thomas J. Oral latex desensitization of healthcare workers. Allergy Clíni Immunol 2001; 101:1: 2: S 161.

2. Pereira C, Rico P, Lourenço M, Lombardero M Pinto-Mendes J, Chieira C. Specific immunotherapy for occupational latex allergy. Allergy 1999; 54: 291-293.

3. Nucera E, Schiavino D, Buonomo A, Roncallo C Del Ninno M, Milani A et al. Latex rush desensitization. Allergy 2001; 56: 86-87.

4. Turjanmaa K, Palosuo T, Alenius H, Leynadier F, Autegarden JE, ANDRÉ C et al. Latex allergy diagnosis: in vivo and in vitro standardization of a natural rubber latex extract. Allergy 1997; 52: 41-50.

5. Leynadier F, Herman D, Vervloet D, Andre C. Specific immunotherapy with a standardized latex extract versus placebo in allergic healthcare workers. J Allergy Clin Immunol 2000; 106: 585-590.

6. MALLING HJ. Immunotherapy as an effective tool in allergy treatment. Allergy 1998; 53 $461-472$

7. Lombardero M, Barber D, Rico P, Wikborg T, SASTRE J. Characterization of latex extract for clinical use. In vitro measurements. J Allergy Clin Immunol 2000; 105: 261-262.

8. Sastre J, Fernández-Nieto M, Rico P, Martin S, Barber D, Cuesta J, las Heras M, Quirce $S$ et al Specific immunotherapy with a standardized latex extract in allergic workers: a doubleblind, placebo-controlled study. J Allergy Clin Immunol 2003; 111: 984-994.

9. Charous Bl, Blanco C, Tarlo S, Hamilton RG, BAUR X et al. Yunginger Natural rubber latex allergy after 12 years: Recommendations and perspectives. J Allergy Clin Immunol 2002 109: 31-34.

10. Patriarca G, Nucera E, Pollastrini E, Roncallo C, Buonomo A, Bartolozzi F et al. Sublingual desensitization: a new aproach to latex allergy problem. Anesth Analg 2002; 95: 956 960.

11. Patriarca G, nucera E, Buonomo A, Roncallo C, De Pasquale T, Pollastrini E et al. New insights on latex allergy diagnosis and treatment. J Investig Allergol Clin Immunol 2002; 12: 169-176.

12. Fernandez M, Sastre J, Quirce S, Cisteró A Enrique E, SAN Miguel MM et al. Inmunoterapia sublingual con un extracto de latex (slit-latex): tolerancia y evolución en la reactividad cutánea. Rev Esp de Alergol 2002; $17: 2$. 